\title{
Validación de un cuestionario sobre la actitud docente frente a la educación inclusiva en Chile*
}

\author{
Validation of a Questionnaire on Teacher Attitudes Towards \\ Inclusive Education in Chile
}
Emilio Rodríguez Macayo** , Francisca González Gil*** , Elena Pastor*** , Rubén Vidal Espinoza $^{* * * * *}$

\begin{abstract}
RESUMEN
La actitud de los docentes frente a la inclusión es un factor clave si queremos avanzar hacia el desafío de contar con comunidades educativas cada vez más inclusivas. El objetivo de este estudio busca validar un cuestionario sobre la actitud docente frente a la inclusión educativa en Chile. Se efectúa un estudio descriptivo survey, de corte transversal-transeccional, no experimental, con una muestra no probabilística (accidental). Para ello se encuesta a 497 docentes que se desempeñan en escuelas municipales de Chile. Se utiliza un cuestionario con escala Lickert con 19 preguntas, para determinar la actitud frente a la inclusión. La medida de Kaiser-Meyer-Olkin KMO fue 0.893 y la prueba de esfericidad de Bartlet fue altamente significativa $(\mathrm{X} 2=4196.261, \mathrm{gl}=171$, sig 0,000$)$. Por otro lado, las cargas factoriales en las 19 preguntas
\end{abstract} Palabras clave: actitud, inclusión, docentes, cuestionario, validación.

Trabajo realizado por el autor principal en el marco del Proyecto FS/22-2017 Evaluación Transcultural de la formación del profesorado para la inclusión: estudio comparativo entre España y Latinoamérica.

"* Máster en Integración social de personas con Discapacidad. Universidad de Salamanca. España. Filiciación Universidad Católica Silva Henríquez. Chile. Nacionalidad Española. Correo electrónico erodriguez@ucsh.cl. N ORCID 0000-0002-1321-2382.

*** Doctora en Educación Universidad de Salamanca-España. Filiación Universidad de Salamanca. España. Nacionalidad Española. Correo electrónico: frang@usal.es. $\mathrm{N}^{\circ} \mathrm{Re}-$ gistro ORCID 0000-0003-1276-7877.

Doctora en Educación Universidad de Salamanca-España. Filiación Universidad de Salamanca. España. Nacionalidad Española. Correo electrónico: emapa@usal.es. Nº Registro ORCID 0000-0002-8046-748.

*n**** Magister en Educación Especial de la Pontificia Universidad Católica. Chile. Filiación Universidad Católica Silva Henríquez. Chile. Correo electrónico: rvidale@ucsh.cl. $\mathrm{N}^{\circ}$ Registro ORCID 0000-0002-8593-5249. 
oscilaron entre $<0.500$ a 0.899 y la fiabilidad en general evidenció un Alpha de Cronbach ( $\mathrm{r}=0.85$ ). Se verifica, por tanto, que el cuestionario es válido y confiable en Chile para determinar la actitud docente frente a la inclusión educativa.

\begin{abstract}
The teacher's attitude towards inclusion is a key factor if we want to address the challenge of constructing increasingly inclusive educational communities. The objective of this study looks to validate a questionnaire on the teacher's attitude towards educational inclusion in Chile. A descriptive study is performed - in this case, a survey - which is transversal-transectional, non-experimental, with a non-probabilistic (accidental) sample. The survey was applied to 497 teachers at public schools in Chile. The questionnaire used the Likert scale and consisted of 19 questions to determine their attitudes towards inclusion. The Kaiser-Meyer-Olkin (KMO) index was 0.893, and the Bartlett sphericity test was highly significant $(\mathrm{X} 2=4196.261, \mathrm{gl}=171$, sig 0.000$)$. On the other hand, the factor loads in the 19 questions varied between $<0.500$ and 0.899 , and the overall reliability yielded a Cronbach's alpha $(\mathrm{r}=0.85)$. It was thus verified that the questionnaire is valid and reliable in Chile to determine teacher attitudes towards educational inclusion.
\end{abstract}

Key words: attitude, inclusion, teachers, questionnaire, validation. 


\section{Introducción}

El plantea del problema de la inclusión surge como consecuencia de los altos índices de exclusión y desigualdades educativas que persisten en la gran mayoría de los sistemas educativos (Blanco, 2006). Es así como el gran desafío de todos los países al año 2030 consiste en garantizar una educación inclusiva, equitativa y de calidad, promoviendo oportunidades de aprendizaje durante toda la vida y para todos. Lo anterior supone que todos los países deben comprometerse a:

Proporcionar una educación de calidad, inclusiva e igualitaria a todos los niveles: enseñanza preescolar, primaria, secundaria, terciaria y formación técnica y profesional. Todas las personas, sea cual sea su sexo, raza u origen étnico, incluidas las personas con discapacidad, los migrantes, los pueblos indígenas, los niños y los jóvenes, especialmente si se encuentran en situaciones de vulnerabilidad, deben tener acceso a posibilidades de aprendizaje permanente, que les ayuden a adquirir los conocimientos y aptitudes necesarios para aprovechar las oportunidades que se les presenten y participar plenamente en la sociedad. (Organización de las Naciones Unidas, ONU, 2015, p. 15).

En el desafío de avanzar hacia una educación para todos, en la Declaración de Salamanca, marco de acción para las necesidades educativas especiales, se ratificó lo planteado en la Declaración Universal de los Derechos Humanos (1990) sobre el derecho que todos los niños, niñas y jóvenes, sin importar su sexo u otras diferencias, tienen a ser educados (Organización de las naciones Unidas para la Educación la Ciencia y la Cultura, UNESCO, 1994).

De la misma forma, la UNESCO en la Declaración de Incheon (2015), reafirma la visión del movimiento mundial en pro de la educación para todos, que se puso en marcha en Jomtien en 1990. Asimismo, ratifica la visión y la voluntad política reflejadas en numerosos tratados de derechos humanos internacionales y regionales en los que se establece el derecho a la educación y su interrelación con otros derechos humanos. En la reunión mundial sobre la Educación para Todos (Mascate, 2014), del mismo modo, declara la preocupación por la persistencia de las desigualdades en cuanto al acceso, la participación y los resultados de aprendizaje en todos los niveles educativos. 
Se hace preponderante que sean los propios sistemas educativos quienes enfrenten sólidamente el desafío de modificar elementos fundamentales, tales como sus culturas, políticas y prácticas educativas, proporcionando a todos los estudiantes las respuestas a sus necesidades, con el objetivo de desarrollar al máximo sus capacidades y, de esta forma, lograr el acceso, participación y progreso bajo el paradigma de la inclusión.

$\mathrm{Al}$ respecto, para Booth y Ainscow (2015), la inclusión no solo se relaciona con eliminar o minimizar las barreras que limitan el aprendizaje y la participación de todo el estudiantado. Los diversos grupos sociales, etnias y culturas, tienen normas, valores, creencias y comportamientos distintos, que generalmente no forman parte de la cultura escolar, lo que puede limitar sus posibilidades de aprendizaje y de participación, o conducir a la exclusión y discriminación.

Las escuelas inclusivas representan un marco favorable para asegurar la igualdad de oportunidades y la plena participación, contribuyendo a una educación más personalizada, que fomente la colaboración entre todos los miembros de la comunidad escolar y constituya un paso esencial para avanzar hacia sociedades más inclusivas y democráticas.

La oferta curricular, la gestión escolar, las estrategias de aprendizaje que se utilizan en el aula, las actitudes de los profesores, entre otros factores, pueden favorecer o dificultar el desarrollo y aprendizaje de los estudiantes y su participación en el proceso educativo. Por ejemplo, un mismo estudiante puede tener dificultades en una escuela y no en otra, dependiendo de cómo se aborde en cada una la diversidad. Por esto la importancia de que las escuelas sean promotoras de la inclusión y, aún más importante, de suprimir actos de exclusión. La escuela, según Booth y Ainscow (2015), tiene, en este aspecto, un papel fundamental para evitar que las diferencias de cualquier tipo se conviertan en desigualdades educativas y, por esa vía, en desigualdades sociales, produciéndose un círculo vicioso difícil de romper. Ainscow (2012) afirma que las escuelas inclusivas pueden ser de diversas formas, pero lo que todas ellas tienen en común es la existencia de una cultura organizativa que contemple la diversidad de los estudiantes de manera positiva.

En la escuela inclusiva es fundamental identificar cuáles son las barreras que producen la exclusión en las aulas y cuáles deberían ser las 
ayudas para reconducir la inclusión. Se entiende por "ayudas" aquellos recursos del contexto educativo que contribuyen a que el estudiantado esté incluido social y educativamente en las aulas. Contrario a esto, las barreras son los obstáculos que dificultan o limitan el aprendizaje, la participación y la convivencia en condiciones de equidad. Para López (2012) el concepto de "barrera" hace referencia a la falta de recursos, de experiencia, y a la existencia de métodos de enseñanza y de actitudes que pudieran limitar la presencia, participación y aprendizaje de determinados estudiantes.

Para el reconocimiento de las barreras en un establecimiento, Booth y Ainscow (2015) ponen a disposición la "Guía para la educación inclusiva: desarrollando el aprendizaje y la participación de los centros escolares", que permite a los establecimientos educativos explorar la posibilidad de la mejora escolar apoyados en tres dimensiones que se encuentran interconectadas: creación de cultura inclusiva, generación de política inclusivas y desarrollo de práctica inclusiva. De ellas es importante resaltar la importancia de la dimensión "creación culturas inclusivas", en cuanto es a partir de las políticas y prácticas que se produce una diferencia en la cultura y hace que esta se mantenga.

En las últimas décadas, en América Latina y en Chile se ha visualizado la desigualdad y exclusión que produce la pobreza y los sistemas ambiguos de clasificación de clases sociales, estableciendo necesidades en el ámbito educativo que la inclusión promete como desafío cubrir. Infante manifiesta que:

El concepto de inclusión ha adquirido un énfasis especial durante los últimos años en el contexto educativo latinoamericano y particularmente el chileno, visibilizándose en ámbitos como políticas públicas y acciones gubernamentales. Esta representación del concepto de inclusión regula no sólo las prácticas educacionales (enseñanza, metodología, currículum, entre otras) sino las ideas sobre situaciones de exclusión, diversidad y, de manera significativa, sobre la construcción de identidades pedagógicas. (2010, p. 288).

En este sentido, Chile ha asumido el gran desafío de transformar su educación, transitando desde un foco centrado en la homogeneización de sus prácticas hacia una educación que respete, valore y aproveche las diferencias individuales presentes en las aulas de clases. 
Lo anterior se manifiesta en la promulgación de la Ley General de Educación $N^{\circ} 20.370 / 2009$ del Ministerio de Educación de Chile, en la que se define la educación como un derecho de todas las personas, reconociéndola como un proceso de aprendizaje permanente, que abarca las distintas etapas de la vida y que tiene como finalidad alcanzar el desarrollo espiritual, ético, moral, afectivo, intelectual, artístico y físico, mediante la transmisión y el cultivo de valores, conocimientos y destrezas.

Es importante destacar que la citada Ley se enmarca en el respeto y valoración de los derechos humanos y de las libertades fundamentales, de la diversidad multicultural y de la paz, y de nuestra identidad nacional. En este desafío, liderado por el Estado de Chile por asegurar el derecho a la educación de todos, la promulgación de la Ley de Inclusión Escolar $N^{\circ}$ 20.845/2015 del Ministerio de Educación viene a impulsar la diversidad de procesos de los distintos ámbitos educativos, desde las diferentes realidades culturales, religiosa-social y las variaciones que puedan existir en los proyectos educativos institucionales.

Ese mismo año se promulga del Decreto $\mathrm{N}^{\circ}$ 83/2015 del Ministerio de Educación de Chile, que viene a orientar a los docentes de los niveles educativos de párvulos y básica para la implementación de procesos de diversificación de la enseñanza, mediante la transición de un modelo centrado en el déficit, a un modelo educativo capaz de atender a todos los estudiantes sin importar sus habilidades, características, necesidades individuales, contexto, entre otros. Por lo anterior la respuesta a la diversidad, en el sentido amplio del término, es inherente a todo proceso educativo y no debiera concebirse como tarea exclusiva de ciertos profesores; por el contrario, debe ser una función de todos los docentes (MINEDUC, 2015). Importante señalar que el Decreto $\mathrm{N}^{\circ}$ 83 establece algunos principios provenientes de un modelo estratégico de enseñanza, como es el Diseño Universal de Aprendizaje (DUA); sin embargo, la población docente de Chile contó con una escasa formación pertinente para la implementación efectiva de este decreto.

Todas las acciones presentadas se orientan hacia la construcción de cambios inclusivos en el ámbito social, advirtiendo la necesidad de que deben considerarse todos y cada uno de los elementos funcionales en el sistema educativo. Sin embargo, estas trasformaciones no generan impacto si no es que van de la mano de un cambio significativo 
en la actitud de los fututos docentes, como de quienes actualmente se encuentran en pleno ejercicio profesional.

En este aspecto, González, Martín y Baz (2017), logran constatar que el éxito de la educación y, por ende, de la educación inclusiva, depende en gran medida de la preparación del profesorado, de sus competencias y actitudes para enseñar en un entorno en el que la diversidad es un aspecto que suma en el proceso de enseñanza-aprendizaje de los estudiantes.

Es interesante indagar que hay detrás de todo este concepto de "actitud" y su vinculación con la educación inclusiva. Al respecto, el concepto de "actitud" engloba diferentes componentes. Para Triandis (1971), viene a ser como una idea cargada de emoción que predispone a una clase de acciones ante un determinado tipo de situaciones sociales. Asimismo, Chinner (2011) la define como la predisposición que afecta consistentemente la respuesta de las personas hacia determinados objetos o situaciones, y considera la percepción como la interpretación personal de la información disponible sobre un determinado dato o fenómeno.

Las actitudes han sido estudiadas desde el punto de vista psicológico por diferentes autores (Vicuña, 2013; Castro, 2016; Castillo, 2016), quienes plantean como tres los componentes que constituyen una actitud: la idea, componente cognitivo; la emoción asociada a ella, componente afectivo, y la predisposición a la acción, componente conductual.

Respecto de la relación entre actitudes e inclusión, González y Martín (2014) señalan que en el contexto escolar las actitudes son predictores de la educación inclusiva y de la respuesta a la diversidad. Influyen directamente en la construcción del conocimiento de los profesores acerca de sus estudiantes, así como en la enseñanza y su propia práctica docente, configurándose de este modo como un aspecto clave en las expectativas que desarrollan en torno a sus estudiantes y sus aprendizajes, y que, al mismo tiempo, se relacionan con el rendimiento académico de éstos.

Más concretamente, según Meijer, (2003), uno de los factores predictores de la aceptación de la diversidad, la construcción del conocimiento de los estudiantes y las prácticas educativas que se manifiestan 
como más incluyentes son precisamente las actitudes docentes. Lo que se relaciona con las expectativas sobre los aprendizajes y el rendimiento académico de los alumnos (Aguado et al., 2008; Chiner 2011; González, 2016). Por otra parte, San Martín y colaboradores (2017) consideran que la formación inicial y continua de los agentes de la educación también se torna parte de este papel crucial en la práctica educativa. En este sentido, el desarrollo de actitudes positivas ante los estudiantes con necesidades educativas especiales forma parte de una de las mayores preocupaciones existentes en la actualidad, principalmente entre docentes.

La inclusión, por tanto, implica un cambio en las actitudes, en la manera de entender la educación y en el modo de responder a las necesidades educativas de todos los estudiantes (Chinner, 2011). Este cambio o transformación requiere que la sociedad modifique su pensamiento, elimine sus prejuicios y logre desarrollar actitudes más positivas hacia las diferencias individuales. En esta misma línea, Sanhueza, Granada y Bravo (2012) consideran que aquellos profesores con actitudes negativas hacia la inclusión tienen además escasas expectativas de logro hacia sus estudiantes, quedando demostrado en la escasa utilización de estrategias que eliminen las barreras y/o las transformen en facilitadores para el aprendizaje. Por lo tanto, los profesores que presentan actitudes negativas para dar respuesta a la diversidad de estudiantes, probablemente no tuvieron una formación inicial pertinente a la temática señalada.

Por otra parte, Álvarez y Buenestado (2015) determinan que la calidad de la educación, para que sea inclusiva, requiere de una formación inicial y continua de los profesores, basada en conocimientos, competencias, valores y actitudes. Sin embargo, la investigación sugiere para que una inclusión sea efectiva y responsable se requiere no solo de buenas disposiciones (valores, creencias, actitudes positivas y profesionalidad), sino también de buenas prácticas; es decir, de un profesorado competente, capaz de atender las necesidades diversas de su alumnado (Chinner, 2011). En la misma línea, Garzón, Clavo y Orgáz manifiestan que:

Las actitudes docentes en las salas de clases pueden convertirse en una barrera o un facilitador en el proceso de enseñanza aprendizaje de todos los estudiantes, evidenciándose en la utilización de un mayor o menor uso de estrategias para la inclusión. (2016, p. 27). 
De esta manera, las actitudes del profesor en relación a los estudiantes con alguna necesidad educativa especial se verán reflejadas en el aula, pudiendo convertirse en uno de los principales obstáculos para llevar a cabo los procesos de inclusión (Damm, 2014). Si bien es cierto, Umesh y Kate (2016) aportan que la mayoría de los profesores poseen actitudes positivas hacia la inclusión, esta depende del nivel de severidad de necesidades educativas especiales que presenten sus estudiantes en su propia aula; de la misma forma, aquellos profesores que cuentan con apoyo por parte de los equipos directivos y otro profesional de apoyo en las aulas, también presentan actitudes positivas hacia la inclusión, comparado con aquellos que carecen de ayudas.

La formación profesional que los docentes hayan recibido acerca de la inclusión educativa es prioritaria en los informes y debates políticos que vienen dándose en las últimas décadas, tales como "Guías para las políticas sobre la inclusión en educación” (UNESCO, 2009); el World Report on Disability (WHO, 2011), el Informe de la Organización para la Cooperación y el Desarrollo Económico (OCDE, 2010) sobre la educación de profesores para la diversidad, y el trabajo de la Agencia Europea para el Desarrollo, acerca de las necesidades de formación del profesorado para la educación inclusiva en Europa (EADSNE, 2012). $\mathrm{Al}$ respecto, existe consenso de que son los docentes y sus acciones los principales protagonistas para llevar a cabo procesos de inclusión educativa, lo que hace urgente que puedan avanzar en la comprensión de lo que creen, lo que pueden hacer y lo que hacen.

La teoría y la práctica de la educación inclusiva, según Martinic (2008), indican que uno de los elementos que más incide en el proceso de aprendizaje de los estudiantes tiene que ver con lo que creen, pueden y están dispuestos a hacer los docentes, y con las expectativas sobre los logros de sus estudiantes. En este contexto, autores como Jordan (2007) y Palomares (2011) entienden que el profesor es agente clave para la construcción de una escuela inclusiva de calidad, al ser $e l$ instrumento pedagógico por excelencia. No es posible avanzar respecto del cambio del modelo institucional si no es a partir del trabajo sobre las representaciones de los profesores, pues la herramienta más potente para el cambio es desde el profesorado y con el profesorado. De no ser así (Sales et al., 2001; Sánchez et al., 2008; Monereo, 2010), las carencias formativas en el profesor sobre atención a la diversidad 
pueden provocar sensaciones de desorientación, vulnerabilidad, incapacidad, desinterés e incluso rechazo.

Asimismo, estudios recogen cómo las actitudes del profesorado dependen en cierta medida de variables como la edad, género, título profesional, nivel educativo, años de experiencia y formación continua. Además, otras investigaciones muestran que el tipo de actitud depende de las características de los estudiantes, de la disponibilidad de recursos, del apoyo y el tiempo disponible, así como de la formación docente y capacitación para enseñar a la diversidad de estudiantes (Garzón et al., 2016).

Por todo lo anterior, y con el objetivo de que todos los estudiantes sean parte del sistema educativo, se hace imperativo que sus profesores rechacen los procesos de exclusión y se comprometan en la inclusión plena de sus alumnos. Por tanto, si los profesores actualmente manifiestan no sentirse preparados para trabajar con todos los estudiantes (Holdheide y Reschly, 2008), el desafío se traslada a mejorar la calidad de los programas de formación -tanto inicial como continuade los profesores, contemplando sus necesidades y actitudes hacia la inclusión educativa.

De acuerdo a lo anterior, expertos chilenos, convocados por el Ministerio de Educación a la Mesa Técnica de Educación Especial (MTEE), "Propuestas para avanzar hacia un sistema educativo inclusivo en Chile: Un aporte desde la Educación Especial” (2015), consideran que para el logro de un sistema educativo inclusivo se requiere la resignificación de la profesión docente y cambios sustantivos en los modelos y el currículo de la formación inicial. La educación inclusiva demanda de los profesionales de la educación, ser sensibles a las diferencias y contar con las competencias necesarias para trabajar en contextos complejos, socioculturalmente diversos y con grupos heterogéneos. De esta forma (UNESCO, 2007), la respuesta a la diversidad implica el tránsito desde un enfoque homogeneizador, en el que se ofrece lo mismo a todos, a un enfoque que considere las distintas identidades, necesidades y opiniones de cada uno.

Finalmente, para González y Martín (2014) la formación inicial de futuros profesionales de la educación en el enfoque de la inclusión es clave para el desarrollo de actitudes positivas frente a estos procesos, 
por ello, un elemento primordial es reconsiderar el currículo en la formación de todos los profesionales que practican la docencia en los distintos países. Por tanto, si los profesores no se sienten preparados para trabajar con todos los estudiantes, el desafío está en mejorar sus programas de formación, a partir de sus propias necesidades docentes y su actitud hacia la educación inclusiva.

\section{Método}

\section{Tipo de estudio y muestra}

Este estudio se realiza desde un enfoque cuantitativo, que utiliza la recolección de datos para probar hipótesis con base en la medición numérica y el análisis estadístico (Hernández et al., 2014), estableciendo como finalidad pautas de comportamiento y comprobación de teorías.

Se aplica el diseño descriptivo que, de acuerdo con Hernández y colaboradores (2014), mide, evalúa y/o recolecta datos sobre diversos conceptos (variables), aspectos, dimensiones o componentes del fenómeno a investigar. Se trató de un estudio no experimental, transeccional (o transversal), caracterizado por investigar en un mismo momento distintos individuos, los cuales representan diferentes etapas del desarrollo.

La técnica de investigación que se desarrolla es de tipo survey, la cual, según Cossio (2015), procura determinar prácticas presentes u opiniones de una población específica, mediante cuestionarios de opinión que se aplican en diferentes contextos.

La muestra está compuesta por 497 docentes que se desempeñan en establecimientos educativos en Chile, impartiendo clases en los distintos niveles educativos en los que se estructura el sistema educativo chileno. La elección de la muestra fue de tipo no probabilístico, en la que la elección de los elementos no depende de la probabilidad, sino de las características de la investigación (Hernández et al., 2014) $y$, por conveniencia, docentes de los distintos niveles educativos, con distintos rangos de edad, con una formación universitaria diferente y con distintos años de experiencia. En la tabla 1 se describe la distribución de la muestra. 
Tabla 1

Distribución de la muestra

\begin{tabular}{|c|c|c|c|c|}
\hline & \multicolumn{4}{|c|}{ Titulo profesional y edad } \\
\hline & $\begin{array}{l}\text { Menor de } \\
30\end{array}$ & $\begin{array}{l}\text { Entre } 31 \\
y 40\end{array}$ & $\begin{array}{l}\text { Entre } 41 \\
\text { y } 50\end{array}$ & $\begin{array}{l}\text { Mayor de } \\
50\end{array}$ \\
\hline Ed. de Párvulos & $12,0 \%$ & $46,0 \%$ & $14,0 \%$ & $28,0 \%$ \\
\hline Profesor Ed. Básica & $24,0 \%$ & $38,6 \%$ & $12,2 \%$ & $25,2 \%$ \\
\hline Profesor Ed. Media & $20,7 \%$ & $31,7 \%$ & $20,7 \%$ & $26,8 \%$ \\
\hline Profesor Ed. Especial & $31,5 \%$ & $46,6 \%$ & $16,4 \%$ & $5,5 \%$ \\
\hline \multirow[t]{3}{*}{ Más de uno } & $18,4 \%$ & $36,8 \%$ & $18,4 \%$ & $26,3 \%$ \\
\hline & \multicolumn{4}{|c|}{ Nivel en que se desempeña y edad } \\
\hline & $\begin{array}{l}\text { Menor de } \\
30\end{array}$ & $\begin{array}{l}\text { Entre } 31 \\
\text { y } 40\end{array}$ & $\begin{array}{l}\text { Entre } 41 \\
\text { y } 50\end{array}$ & $\begin{array}{l}\text { Mayor de } \\
50\end{array}$ \\
\hline Ed. de Párvulos & $14,8 \%$ & $48,1 \%$ & $13,0 \%$ & $24,1 \%$ \\
\hline Profesor Ed. Básica & $24,0 \%$ & $39,8 \%$ & $13,8 \%$ & $22,4 \%$ \\
\hline Profesor Ed. Media & $14,1 \%$ & $35,9 \%$ & $21,8 \%$ & $28,2 \%$ \\
\hline \multirow[t]{3}{*}{ Más de uno } & $36,1 \%$ & $32,8 \%$ & $13,1 \%$ & $18,0 \%$ \\
\hline & \multicolumn{4}{|c|}{ Años de experiencia y edad } \\
\hline & $\begin{array}{l}\text { Menor de } \\
30\end{array}$ & $\begin{array}{l}\text { Entre } 31 \\
y 40\end{array}$ & $\begin{array}{l}\text { Entre } 41 \\
\text { y } 50\end{array}$ & $\begin{array}{l}\text { Mayor de } \\
50\end{array}$ \\
\hline 5 o menos & $70,3 \%$ & $29,1 \%$ & $0,7 \%$ & $0,0 \%$ \\
\hline Entre 6 a 10 años & $7,0 \%$ & $78,9 \%$ & $10,9 \%$ & $3,1 \%$ \\
\hline Entre 11 a 15 años & $1,3 \%$ & $55,3 \%$ & $38,2 \%$ & $5,3 \%$ \\
\hline Entre 16 a 20 años & $0,0 \%$ & $26,5 \%$ & $41,2 \%$ & $32,4 \%$ \\
\hline Más de 20 años & $0,0 \%$ & $0,0 \%$ & $14,4 \%$ & $85,6 \%$ \\
\hline
\end{tabular}

Elaboración propia, 2020.

\section{Técnicas y procedimientos}

La técnica utilizada es la encuesta y el instrumento de recolección es un cuestionario de escala tipo Likert denominado: "Cuestionario de valoración docente de la inclusión educativa. CEFI-R, de González y Martín (2014). Este instrumento fue adaptado en su lenguaje para ser aplicado en Chile, y sometido a validación por juicio expertos a través del estudio "Actitud y necesidades formativas frente a la inclusión de 
docentes de la provincia de Talca-Chile", llevado a cabo por Núñez y Rojas (2017) en el marco de su proceso de tesis conducente al grado académico de Magíster en Gestión Inclusiva, realizado en la Universidad Santo Tomás de la ciudad de Talca, Chile.

El cuestionario está formado por 19 ítems que miden las actitudes, competencias, habilidades y destrezas docentes con base en cuatro factores que se agrupan en tres dimensiones: Dimensión crear Culturas Inclusivas, Dimensión generar Políticas Inclusivas y Dimensión desarrollar Políticas Inclusivas.

Respecto de lo anterior, se señala que la Dimensión crear Cultura Inclusiva se compone de dos factores: Concepción de la Diversidad y Participación de la Comunidad. En relación con el primero, este recoge la valoración personal sobre el concepto de "diversidad", el lugar y la forma en que se contempla la escolarización de los estudiantes, la política educativa en la que se sustentan estas decisiones y, en definitiva, la interpretación individual de la educación inclusiva. El segundo factor, relativo a la Participación de la Comunidad, se orienta hacia la recogida de información sobre el trabajo colaborativo entre el centro educativo y el resto de agentes de la comunidad, así como del uso de los recursos que, más allá del recinto de la escuela, nos ofrece el entorno.

En la misma línea anterior, la Dimensión generar Política Inclusiva contiene el Factor Apoyos, que recoge información en torno al concepto de "apoyo", el rol que debe desempeñar el profesor de apoyo, los potenciales destinatarios de dicho apoyo y el lugar donde se proporciona y, por tanto, el trabajo colaborativo entre este profesional y el resto de los profesores.

Finalmente, en la Dimensión desarrollar Prácticas Inclusivas se aloja el Factor Metodología, el cual contempla la perspectiva desde la que se define cada uno de los elementos que forman parte del currículo, como son las estrategias metodológicas, recursos, materiales, técnicas de comunicación, evaluación entre otros. Del mismo modo, contempla si los docentes se consideran preparados para implementar los elementos del currículo desde la óptica de la inclusión.

El instrumento se aplicó a todos los docentes seleccionados usando método tradicional, papel y lápiz, contando con el tiempo requeri- 
do para aquello. Contestaron todos los docentes seleccionados y que asistieron el día de la aplicación, firmando a su vez un consentimiento informado que garantizó la confidencialidad, el anonimato y el uso de la información resultante únicamente con fines investigativos. Del mismo modo, no contestaron el cuestionario aquellos docentes que no asistieron y no firmaron consentimiento informado.

\section{Resultados}

El cuestionario CEFI-R, fue validado por el método de validez de constructo. Se aplicó un análisis factorial exploratorio (Bandalos \& Finney, 2010; Hoyle \& Duvall, 2004) para analizar la estructura. Una vez efectuada la rotación de los resultados, se han convergido en tres factores. El grado de confiabilidad se determinó por medio de consistencia interna, Alpha de Cronbach, cuyo resultado es de 0.85 .

El primer paso consistió en verificar la significancia del conjunto de reactivos para proceder al análisis factorial por medio de la KaiserMeyer-Olkin Measure (KMO), que tuvo un valor de 0.893, con una esfericidad de la prueba de Bartlett's de $\mathrm{X}^{2}=4196.261$, $\mathrm{gl}=171$, sig 0,000, valores que son interpretados como favorables para continuar con la validez del instrumento y el análisis factorial.

La estructura factorial del cuestionario se evaluó mediante la opción del método de componentes principales y rotación varimax, y una extracción con el método de Máxima Verosimilitud, estableciendo un puntaje de corte de .50. Para este paso se introdujeron los 19 ítems distribuidos en 4 factores del cuestionario original, teniendo como resultado que solamente 11 de ellos muestran una carga factorial $(<0.50)$, tal y como se muestran agrupados en la tabla 2 . 
Tabla 2

Matriz factor rotado

\begin{tabular}{lccc}
\hline & $\begin{array}{c}\text { Dimensión 1 } \\
\text { Crear Cultura In- } \\
\text { clusiva }\end{array}$ & $\begin{array}{c}\text { Dimensión 2 } \\
\text { Generar Política } \\
\text { Inclusiva }\end{array}$ & $\begin{array}{c}\text { Dimensión 3 } \\
\text { Desarrollar Práctica } \\
\text { Inclusiva }\end{array}$ \\
\hline P4 & 0,899 & & \\
P5 & 0,850 & & \\
P3 & 0,840 & & \\
P2 & 0,677 & 0,849 & \\
M3 & & 0,813 & \\
M4 & & 0,656 & \\
M2 & & 0,507 & \\
M5 & & & 0,728 \\
A2 & & 0,538 \\
A4 & & & 0,526 \\
A1 & & & \\
\hline
\end{tabular}

Elaboración propia.

Las cargas factoriales en las tres dimensiones oscilaron entre $<0.50$ y 0.899 , y el resultado de la prueba de consistencia interna del cuestionario con 19 ítems.

Todos los ítems que componían el Factor Conocimiento de la Diversidad (C1: Preferiría no tener en mi aula estudiantes con necesidades educativas especiales; C2: Un estudiante con necesidades educativas especiales interrumpe la rutina del aula y perjudica el aprendizaje de sus compañeros; C3: No debemos escolarizar estudiantes con necesidades educativas especiales derivadas de una discapacidad en establecimientos regulares hasta que no tengamos la formación adecuada para ello; C4: Los estudiantes con necesidades educativas especiales no pueden seguir la planificación clase a clase y C5: Me preocupa que mi carga de trabajo se incremente si tengo estudiantes con necesidades educativas especiales en mi clase) y el ítem P1: El proyecto educativo institucional (PEI) debería revisarse con la participación de los distintos agentes de la comunidad educativa (docentes, padres, estudiantes...) del Factor Participación de la Comunidad ambos de la Dimensión Crear Cultura Inclusiva presentan cargas factoriales (>.50), de igual manera ocurre con el ítem Ml: Sé cómo enseñar a cada uno de 
mis estudiantes de manera diferente en función de sus características individuales, del Factor Metodología de la Dimensión desarrollar Prácticas Inclusivas, y el ítem A3: La función del profesional de apoyo es trabajar con todo el alumnado de mi sala de clases, del Factor Apoyos de la Dimensión generar Política Inclusiva.

Se somete el cuestionario con los 11 ítems de mayor carga factorial a la prueba de consistencia interna, mediante el coeficiente de Alpha de Cronbach, y se obtiene un resultado de 0.86 lo que demuestra una mayor consistencia interna y una fiabilidad excelente.

Finalmente, en la tabla 3 se muestra el análisis por dimensión que compone el instrumento, tomando como base los ítems que el análisis factorial entregó con mayor significación (11 de 19).

Tabla 3

Análisis por dimensión de la escala

\begin{tabular}{|c|c|c|c|c|c|c|}
\hline & & Mín. & Máx. & $\mathrm{X}$ & D.E. & C.F \\
\hline \multirow{4}{*}{ 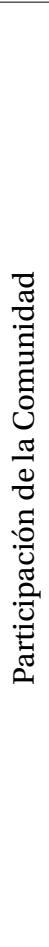 } & $\begin{array}{l}\text { P4: Cada miembro del } \\
\text { establecimiento educativo } \\
\text { (docentes, padres, estudiantes, } \\
\text { otros profesionales) es un } \\
\text { elemento fundamental del } \\
\text { mismo. }\end{array}$ & 1 & 4 & 3,89 & 0,51 & 0,90 \\
\hline & $\begin{array}{l}\text { P5: El establecimiento debe } \\
\text { trabajar de forma conjunta } \\
\text { con los recursos del contexto } \\
\text { comunitario (biblioteca, } \\
\text { servicios sociales, servicio de } \\
\text { salud, ONG). }\end{array}$ & 1 & 4 & 3,81 & 0,55 & 0,85 \\
\hline & $\begin{array}{l}\text { P3: El establecimiento } \\
\text { educativo debe fomentar la } \\
\text { participación de los padres y } \\
\text { apoderados de la comunidad. }\end{array}$ & 1 & 4 & 3,83 & 0,55 & 0,84 \\
\hline & $\begin{array}{l}\text { P2: Es fundamental que haya } \\
\text { una relación muy estrecha } \\
\text { entre los docentes y el resto } \\
\text { de los agentes educativos } \\
\text { (Asistentes de la educación, } \\
\text { centro general de padres y } \\
\text { apoderados, consejo escolar, } \\
\text { juntas vecinales) }\end{array}$ & 1 & 4 & 3,76 & 0,59 & 0,68 \\
\hline
\end{tabular}




\begin{tabular}{|c|c|c|c|c|c|c|}
\hline \multirow{4}{*}{ 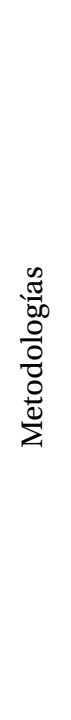 } & $\begin{array}{l}\text { M3: Sé cómo adaptar mi forma } \\
\text { de evaluar a las necesidades } \\
\text { individuales de cada uno de } \\
\text { mis estudiantes. }\end{array}$ & 1 & 4 & 3,24 & 0,81 & 0,85 \\
\hline & $\begin{array}{l}\text { M4: Sé cómo manejar y } \\
\text { adaptar los materiales } \\
\text { didácticos para responder a las } \\
\text { necesidades de cada uno de mis } \\
\text { estudiantes. }\end{array}$ & 1 & 4 & 3,24 & 0,80 & 0,81 \\
\hline & $\begin{array}{l}\text { M2: Sé cómo planificar las } \\
\text { unidades didácticas teniendo } \\
\text { presente la diversidad de los } \\
\text { estudiantes. }\end{array}$ & 1 & 4 & 3,20 & 0,80 & 0,66 \\
\hline & $\begin{array}{l}\text { M5: Soy capaz de adaptar mis } \\
\text { instrucciones para asegurarme } \\
\text { que todos los estudiantes } \\
\text { puedan ser incluidos con éxito } \\
\text { en el aula. }\end{array}$ & 1 & 4 & 3,49 & 0,70 & 0,51 \\
\hline \multirow{3}{*}{$\begin{array}{l}\infty \\
0 \\
0 \\
0 \\
2\end{array}$} & $\begin{array}{l}\text { A2: Creo que la mejor manera } \\
\text { de proporcionar apoyos a los } \\
\text { estudiantes es que el profesional } \\
\text { de apoyo se incorpore también } \\
\text { al aula, en lugar de hacerlo } \\
\text { solamente en el aula de recursos. }\end{array}$ & 1 & 4 & 3,64 & 0,71 & 0,73 \\
\hline & $\begin{array}{l}\text { A4: Considero que el lugar } \\
\text { del profesional de apoyo está } \\
\text { dentro del aula común con } \\
\text { cada uno de los docentes de las } \\
\text { asignaturas. }\end{array}$ & 1 & 4 & 3,43 & 0,82 & 0,54 \\
\hline & $\begin{array}{l}\text { A1: La planificación conjunta } \\
\text { entre docente regular - } \\
\text { profesional de apoyo (en } \\
\text { adelante Profesor/a de } \\
\text { Educadora Diferencial y/o } \\
\text { Psicopedagoga) facilitaria que } \\
\text { los apoyos se proporcionaran } \\
\text { dentro del aula. }\end{array}$ & 1 & 4 & 3,69 & 0,67 & 0,53 \\
\hline
\end{tabular}

Elaboración propia. Nota: Min. = Ptje mínimo; Máx. = Ptje. Máximo; $\mathrm{X}=$ Media; D.E. = Desviación Estándar; C.F. = Carga Factorial.

\section{Discusión y conclusiones}

El objetivo de este trabajo es la validación al contexto chileno de un "Cuestionario de valoración docente de la inclusión educativa" CEFI- 
R (González et al., 2014), que permita evaluar la actitud de docentes chilenos frente de la inclusión educativa.

En las diferentes pruebas a las que fue sometido el instrumento -adaptación al contexto, validación juicio expertos, tratamiento estadístico adecuación muestral, esfericidad de Bartlett, cálculo del Coeficiente de Cronbach, Análisis Factorial, y la correlación interna de los componentes- se presentaron resultados aceptables que evidencian su fortaleza.

Se puede afirmar que el instrumento es válido y fiable para determinar la actitud de docentes chilenos frente a la inclusión. Los resultados dan cuenta de un instrumento de 11 ítems que permiten medir actitudes, competencias, habilidades y destrezas docentes frente a los procesos de inclusión, y, de igual forma, determinar sus necesidades formativas con base en tres factores: Apoyos, Metodología y Participación de la Comunidad.

De manera global, el instrumento, con sus 11 ítems, presentó un Alpha de Cronbach de (r: 0.86) ligeramente superior a la versión original con 19 items (r: 0.85).

El Factor Apoyos aborda el concepto de "apoyo", el rol que debe desempeñar el profesor de apoyo, los potenciales destinatarios de dicho apoyo y el lugar donde se proporciona, y, por tanto, el trabajo colaborativo entre este profesional y el resto de profesores. El Factor Metodología contempla la perspectiva desde la que se define cada uno de los elementos que forman parte del currículo (estrategias metodológicas, recursos, materiales, técnicas de comunicación, evaluación) y si los estudiantes se consideran preparados para realizarlo desde la óptica de la inclusión. El factor Participación de la Comunidad recoge información sobre el trabajo colaborativo entre la escuela y el resto de los agentes de la comunidad, así como del uso de los recursos que, más allá del contexto de la escuela, nos ofrece el entorno.

En el análisis de las dimensiones con cada uno de sus factores asociados, es significativo destacar que los ítems del Factor Conocimiento de la Diversidad de la Dimensión crear Cultura Inclusivas son irrelevantes y no entregan la información esperada, es decir, ser capaces de recoger la valoración personal que cada docente tiene sobre el concepto de "diversidad", el lugar y la forma en que se contempla la escolari- 
zación de los estudiantes, la política educativa en la que se sustentan estas decisiones y, en definitiva, la interpretación individual de la educación inclusiva.

Todo ello nos sitúa en la necesidad de continuar indagando en el contenido de los ítems que definen esta dimensión y su influencia en la composición de la escala, de esta forma, depurarla y crear nuevos ítems que se ajusten a sus destinatarios.

De acuerdo con las puntuaciones obtenidas en cada factor, se pone de manifiesto que los docentes se sienten menos competentes metodológicamente para trabajar desde una perspectiva inclusiva y, de esta forma, proporcionar a sus estudiantes la respuesta que requieren las necesidades que presentan. Es así como, de acuerdo con la información recopilada por medio de los ítems que definen este aspecto, se puede determinar que los docentes presentan carencias formativas en la práctica educativa diaria, lo que se manifiesta en falta de conocimiento en cómo elaborar unidades de aprendizaje, cómo adaptar los materiales didácticos, cómo evaluar, o el uso de técnicas de comunicación diversas.

Lo anterior nos hacer suponer que muchos de los docentes que hoy se encuentran en ejercicio docente no tuvieron una formación inicial que abordara estas temáticas y, junto con ello, que las posibles capacitaciones posteriores no se ajustaron a sus verdaderas necesidades.

Si queremos alcanzar con éxito uno de los principales retos de la agenda 2030, que apunta a la transformación de la educación para que ésta sea abierta a todos, inclusiva y de buena calidad, debemos poner la mirada en los procesos de formación inicial docente. Esto unido a que el éxito de la educación y, por ende, de la inclusión, dependen en gran medida de la preparación de los docentes, de sus competencias y actitudes para enseñar en un entorno donde la diversidad es un aspecto que suma en el proceso de enseñanza-aprendizaje de los estudiantes. Ello nos sitúa ante la necesidad de considerar en el proceso de formación inicial docente de los futuros profesores la inclusión y la educación de la diversidad como un eje transversal, con estrategias de enseñanza y evaluación tendientes a desarrollar competencias asociadas al trabajo colaborativo, la codocencia y, especialmente, a la planificación diversificada. 
Para conocer las competencias que los estudiantes en proceso de formación inicial docente adquieren respecto de la cultura, política y práctica educativa inclusiva, se propone que este cuestionario sea aplicado y validado en estudiantes que se encuentren en su último año de ese periodo, de manera que los resultados sean un aporte al diseño de los planes de estudios de cada uno de los programas de formación de los futuros profesionales de la educación.

\section{Referencias}

Agencia Europea para las Necesidades Educativas Especiales y la Inclusión Educativa. (2012). Perfil profesional del docente en la educación inclusiva. Bruselas.

Aguado, A, Alcedo, Á. \& Arias, B (2008). Cambio de actitudes hacia la discapacidad con escolares de Primaria. Psicothema, 20(4), 697-704. Recuperado de http://www.psicothema.com / pdf/3543.pdf

Ainscow, M. (2012). Haciendo que las escuelas sean más inclusivas: lecciones a partir del análisis de la investigación internacional. Revista de Educación Inclusiva, 5(1), 39-49. Recuperado de https://revistaeducacioninclusiva.es/index.php/REI/article/view/220

Álvarez, J. \& Buenestado, M. (2015). Predictores de las actitudes hacia la inclusión de alumnado con necesidades educativas especiales en futuros profesionales de la educación, Revista Complutense de Educación, XVI(3), 627-645. DOI: https:// doi.org/10.5209/rev_RCED.2015.v26.n3.44551 .

Bandalos, D, \& Finney, S. (2010). Factor analysis: exploratory and confirmatory, en G. Hancock y R. Mueller (Coords.), The reviewer's guide to quantitative methods in the social sciences (pp. 93-114), vol. I.

Blanco, R. (2006). La inclusión en educación: una cuestión de justicia y de igualdad. Revista Electrónica Sinéctica, (29), 19-27. Recuperado de http://www.redalyc.org/pdf/ 998/99815739003.pdf

Booth, T. y Ainscow, M. (2015). Guía para la inclusión educativa: desarrollando el aprendizaje y la participación en las escuelas. Organización de Estados Ibeoramericanos. Recuperado de: http://www.oei.es/historico/publicaciones/detalle_publicacion.php? id $=151$ 
Castillo, C. (2016). Expectativas docentes frente al aprendizaje de niños y niñas con NEE: estudio formulado en una escuela municipal de excelencia. Tesis de Grado Magister, Santiago, Universidad Academia de Humanismo Cristiano-Chile.

Castro, J. (2017). La formación inicial docente en Chile: una parte de su historia y los desafíos de aprendizaje por competencias. Praxis Educativa, 21(2), 12-21. Recuperado de: http://www.redalyc.org/articulo.oa?id=153153528002

Chiner, E. (2011). Las percepciones y actitudes del profesorado hacia la inclusión del alumnado con necesidades educativas especiales como indicadores del uso de prácticas educativas inclusivas en el aula. Tesis de Doctorado, Universidad de Alicante. Recuperado de https://rua.ua.es/dspace/bitstream /10045/19467/1/Tesis_Chiner.pdf

Cossio, M. (2015). Métodos de investigación cuantitativa en ciencias de la educación. Chile: Ediciones Universidad Católica del Maule de Chile.

Damm, X. (2014). Representaciones y actitudes del profesorado frente a la integración de niños/as con necesidades educativas especiales al aula común. Revista Latinoamericana de Educación Inclusiva, 3(1), 25-35. Recuperado de https://sid.usal. es/idocs/F8/ART11920/ representaciones_y_actitudes.pdf

Garzón, P., Calvo, I. \& Orgáz, B. (2016). Inclusión educativa. Actitudes y estrategias del profesorado. Revista Española de Discapacidad, 4(2), 25-45. DOI: https://doi.org/10.5569/23405104.04.02.02

González, F. \& Martín, E. (2014). Educación para todos: formación docente, género y atención a la diversidad. Cuestiones de género: de la igualdad y la diferencia, (9), 11-28. DOI: http:// dx.doi.org/10.18002/cg.v0i9.1151

González, F., Martín, E., Poy, R. \& Jenaro, C. (2016). Percepciones del profesorado sobre la inclusión: estudio preliminar. Revista Electrónica Interuniversitaria de Formación del Profesorado, 19(3), 11-24. DOI: http://dx.doi.org/10.6018/reifop.19.3.219321

Hernández, R., Fernández, C. y Baptista, P. (2014). Metodología de la investigación. Mc Graw Hill.

Hoyle, R. \& Duvall, J. (2004). Determining the number of factors in exploratory and confirmatory factor analysis, in D. Kaplan (Co- 
ord.), The sage handbook of quantitative methodology for social sciences (pp. 301-315), vol. I.

Holdheide, L. \& Reschly, D. (2008). Teacher preparation to deliver inclusive services to students with disabilities. National Comprehensive Center for Teacher Quality, 1-28. Recuperado de https://eric.ed.gov/?id=ED543818

Infante, M. (2010). Desafíos a la formación docente: inclusión educativa. Revista Estudios Pedagógicos, 36(1), 287-297. DOI: http:// dx.doi.org/10.4067/S0718-07052010000100016

Jordán, J. (2007). Educar en la convivencia en contextos multiculturales, en E. Soriano (Coord.) (2007). Educación para la convivencia intercultural (pp. 59-98). La Muralla, (1).

López, J. (2012). Facilitadores de la inclusión. Revista de Educación inclusiva, 5(1), 39-49. Recuperado de https://file.Dialnet-FacilitadoresDeLaInclusion-4105349.pdf

Martinic, S. (2008). Información, participación y enfoque de derechos, en H. Valdés (coord.), Reflexiones en torno a la calidad educativa, Santiago: Laboratorio Latinoamericano de Evaluación de la Calidad de la Educación (13-34), vol I.

Ministerio de Educación de Chile, MINEDUC. (2009). Ley General 20.370/2009, 12 de septiembre, Ministerio de Educación.

Ministerio de Educación de Chile, MINEDUC. (2015). Ley Orgánica 20.845/2015, 8 de junio, Ministerio de Educación.

Ministerio de Educación de Chile, MINEDUC. (2015) Decreto $N^{\circ} 83$. Aprueba criterios y orientaciones de adecuación curricular para estudiantes con necesidades educativas especiales de educación parvularia y educación básica. División educación general, unidad curriculum. Recuperado de http://especial.mineduc.cl/wp-content/uploads/sites/31/2016/08/ Decreto-83-2015.pdf

Ministerio de Educación de Chile, MINEDUC. (2015). Propuesta para avanzar hacia un sistema educativo inclusivo en Chile: un aporte desde la Educación Especial. Recuperado de http://portales.mineduc.cl/usuarios/edu.especial/ doc/201505141109250.INFORMEFINALMESATECNICAEDESPECIAL.pdf

Monereo, C. (2010). La formación del profesorado: una pauta para el análisis e intervención a través de incidentes crítico. Revista 
Iberoamericana de Educación, (52), 149-178. DOI: https:// doi.org/10.35362/rie520615

Núñez, F., Rodríguez, E. y Zambrano, A. (2017). Actitudes y necesidades formativas de los docentes respecto a las culturas, políticas y prácticas educativas inclusivas en la provincia de Talca (Tesis no publicada para optar al grado de Magister en Educación mención Gestión Inclusiva). Talca, Chile: Universidad Santo Tomás.

Organización de las Naciones Unidas para la Educación, la Ciencia y la Cultura, UNESCO. (1994). Declaración de Salamanca y marco de acción ante las necesidades educativas especiales. Salamanca.

Organización de las Naciones Unidas para la Educación, la Ciencia y la Cultura, UNESCO. (2007). Educación de calidad para todos: un asunto de derechos humanos. Documento de educación sobre políticas educativas en el marco de la II reunión intergubernamental del proyecto regional de educación para América Latina y el Caribe. Recuperado de http://unesdoc. unesco.org/ images/0015/001502/150272s.pdf

Organización de las Naciones Unidas para la Educación, la Ciencia y la Cultura, UNESCO. (2009). Policy guidelines on inclusion in education.

Organización de las Naciones Unidas para la Educación, la Ciencia y la Cultura, UNESCO. (2015). Educación 2030: Declaración de Incheon y marco de acción para la realización del objetivo de desarrollo sostenible. Recuperado de http://www.unesco. org/new/fileadmin/ MULTIMEDIA/FIELD/Santiago/pdf/ ESP-Marco-de-Accion-E2030-aprobado.pdf

Organización para la Cooperación y el Desarrollo Económicos, OCDE. (2010). Educating teachers for diversity: meeting the challenge. Recuperado de http://www.oecd.org/education/ ceri/44853338.pdf

Organización de Naciones Unidas, ONU. (2014). Reunión mundial sobre la educación para todos: Declaración final de la reunión mundial sobre la EPT de 2014 el Acuerdo de Mascate. Recuperado de http://www.unesco.org/new/fileadmin/MULTIMEDIA/FIELD/Santiago/pdf/ Muscat-Agreement-ESP.pdf

Organización de Naciones Unidas, ONU. (2015). Trasformar nuestro mundo: "La Agenda 2030 para el Desarrollo Sostenible". Re- 
cuperado de http://unctad.org/meetings/es/Sessional Documents/ares70d1_es.pdf

Palomares, A. (2011). El modelo docente universitario y el uso de nuevas metodologías en la enseñanza, aprendizaje y evaluación. Revista de Educación, (155), 591-604. Recuperado de http:// www.revistaeducacion.educacion.es/re355/re355_25.pdf

Sales, A., Sanchiz, M. \& Moliner, M. (2001). Actitudes hacia la atención a la diversidad en la formación inicial del profesorado. Revista Electrónica Interuniversitaria de Formación del profesorado, 4(2), 39-48. Recuperado de http://web.archive.org/web/ 20041221204450/www.aufop.org/publica/reifp/articulo. asp?pid $=207 \&$ docid $=1026$

San Martín, C., Villalobos, C., Muñoz, C. \& Wyman, I. (2017). Formación inicial docente para la educación inclusiva. Análisis de tres programas chilenos de pedagogía en educación básica que incorporan la perspectiva de la Educación Inclusiva. Calidad en la Educación, (46), 20-52. DOI: http://dx.doi. org/10.31619/caledu.n46.2

Sánchez, A., Díaz C., Sanhueza, S. \& Friz. M. (2008). Percepciones y actitudes de los estudiantes de pedagogía hacia la inclusión educativa. Estudios pedagógicos, 34(2), 169-178. DOI: http:// dx.doi.org/10.4067/S0718-07052008000200010

Sanhueza, S., Granada, M. y Bravo, L. (2012). Actitudes del profesorado de Chile y Costa Rica hacia la inclusión educativa. Cadernos de Pesquisa, 42(147), 884-894. DOI: https://doi.org/10.1590/ S0100-15742012000300013.

Sharma, U. \& Jacobs, K. (2016). Predicting in-service educators' intentions to teach in inclusive classrooms in India and Australia. Teaching and Teacher Education, (55), 13-23. DOI: http:// dx.doi.org/10.1016/j.tate.2015.12.004

Triandis, H. (1971). Attitude and Attitude change. Villey.

Vicuña, K. (2013). Percepciones y actitudes de profesores de primero a séptimo año de educación general básica del distrito educativo 4 hacia la inclusión del alumnado con necesidades educativas especiales como indicadores de uno de prácticas educativas inclusivas en el aula. Tesis de Maestría. Universidad Casa Grande, Ecuador.

World Health Organization, WHO. (2011). World report on disability. Ginebra: WHO. 